\title{
Bootstrap Approaches to Autoregressive Model on Exchange Rates Currency
}

\author{
Muhamad Safiih Lola, Anthea David, Nurul Hila Zainuddin \\ School of Informatics and Applied Mathematics, Universiti Malaysia Terengganu, Kuala Nerus, Malaysia \\ Email: safiihmd@umt.edu.my, ann_peace87@yahoo.com,hila.zainuddin@gmail.com
}

How to cite this paper: Lola, M.S., David, A. and Zainuddin, N.H. (2016) Bootstrap Approaches to Autoregressive Model on Exchange Rates Currency. Open Journal of Statistics, 6, 1010-1024.

http://dx.doi.org/10.4236/ojs.2016.66081

Received: September 10, 2016

Accepted: November 13, 2016

Published: November 17, 2016

Copyright $\odot 2016$ by authors and Scientific Research Publishing Inc. This work is licensed under the Creative Commons Attribution International License (CC BY 4.0).

http://creativecommons.org/licenses/by/4.0/ (c) (i) Open Access

\begin{abstract}
The use of historical data is important in making the predictions, for instance in the exchange rate. However, in the construction of a model, extreme data or dirtiness of data is inevitable. In this study, AR model is used with the exchange rate historical data (January 2007 until December 2007) for USD/MYR and is divided into 1-, 3and 6-horizontal months respectively. Since the presence of extreme data will affect the accuracy of the results obtained in a prediction. Therefore, to obtain a more accurate prediction results, the bootstrap approach was implemented by hybrid with AR model coins as the Bootstrap Autoregressive model (BAR). The effectiveness of the proposed model is investigated by comparing the existing and the proposed model through the statistical performance methods which are RMSE, MAE and MAD. The comparison involves $1 \%, 5 \%$ and $10 \%$ for each horizontal month. The results showed that the BAR model performed better than the AR model in terms of sensitivity to extreme data, the accuracy of forecasting models, efficiency and predictability of the model prediction. In conclusion, bootstrap method can alleviate the sensitivity of the model to the extreme data, thereby improving the accuracy of forecasting model which also have high prediction efficiency and that can increase the predictability of the model.
\end{abstract}

\section{Keywords}

Autoregressive Model, Outliers, Bootstrap, Robust

\section{Introduction}

One of an important aspect in the financial system is the exchange rate. The exchange rate is a rate at which an order currency converted at each other, in addition also referred to as rates of exchange or exchange rates or foreign exchange rate currency arrangement. [1], states that the exchange rate is a price. In contrary, the term price itself 
is the exchange rate. For example, the exchange rate for 102 Japanese yen (JPY, $¥$ ) to 1 US Dollar (USD, \$) denotes the value of $102 \mathrm{JPY}$ is equal to 1 USD. The foreign exchange market is one of the largest markets in the world. In some estimation, about 2 trillion USD value of the order currency changing hands each day.

The Bretton-Woods system of monetary management was introduced in July 1944. However, the system failed in 1971. Since then, much attention has been given to study and forecasts on monetary management. For instance, forecasting the monetary policy in order to maintain the exchange rate. Generally, the exchange rates have a relationship with business in the financial markets. Therefore, it is important for every businessman, corporate members, academicians and anyone related to be aware of forecasting exchange rates.

However, there are constraints that can reduce the accuracy of the prediction in the next set of data that can affect the accuracy of the prediction. Outliers in data are one of the constraints that often cause a high rate of inaccuracy in forecasting. In statistics, outliers are also known as extreme data which is an observation of far deviated from the other data. According to [2] [3], outliers are an isolated observation or external elements which are visible and clearly deviating from the other members in the sample where it occurs. This will result in the inaccurate an unreliable forecasting of future earnings. Outliers can lead to biased parameter estimates and increase the estimated series, so as to damage the quality of the forecasting of the sample. As a result, an Autoregressive (AR) model approach for forecasting has inevitable limitations in predicting the exchange rate returns.

The presence of outliers in the data indeed gives a significant impact on currency exchange rate. From the problems encountered, it is visibly important to establish an approach that can reduce and subsequently solve the problem. The presence of outliers will lead to a high sensitivity to the forecasting models. The consequence of this high sensitivity is that it produces less accurate results, thus providing wrong conclusions. The accuracy of forecasting is important to identify the efficiency of forecasting models used. An efficient forecasting model will in turn increase the predictability of the model. Thus, bootstrap approach will be hybridized to the AR model in this study of exchange rate currency.

Bootstrap approach which is used in this study gives significant impact on exchange rates currency. The method eventually could reduce the effect of outliers as well as provide a more accurate estimation by bootstrap procedure of sampling with replacements. The hybrid between an AR model with bootstrap approach will form a model known as BAR model. BAR models that are less sensitive to outliers allows high predictability of exchange rates currencies.

The high predictability also enables higher forecast accuracy of future exchange rates. According to [4], the accuracy of future forecasting particularly in currency is depend on the value of the present exchange rate, currency, which underlies the value of exchange rates, currency that will be applied in the future. For the bootstrap method, each sampling has the same sample size, $n$ as the original sample. This condition could indi- 
rectly alleviate the problem of outliers in the studied field, such as finance. This in turn reinforces the accuracy of the BAR in the exchange rates currency forecast. In addition, bootstrap approach to AR model also adds the treasury in modeling, especially in the field of AR modeling.

From the proposed model, five objectives which are divided to twofold were highlighted where the first two objectives are tested for accuracy and sensitivity of the model. While the last three are more for the determination of the efficiency, forecasting predictability as well as the appropriate forecasting model. The five objectives of the study are firstly to test the accuracy of the forecasting of USD/MYR exchange rates, currency in 1-, 3- and 6-horizontal months. Secondly, is to test the sensitivity of the model with $1 \%, 5 \%$ and $10 \%$ outliers respectively in 1-, 3- and 6-horizontal months. The next objective is to determine the efficiency of the constructed forecasting models based on the statistical forecasting values obtained at 1-, 3- and 6-horizontal months. The fourth objective is to determine the forecasting predictability for USD/MYR exchange rates, currency in 1-, 3- and 6-horizontal months. While, the last objective is to determine the most appropriate forecasting model for USD/MYR exchange rates, currency based on forecasting accuracy, sensitivity to outliers, forecasting efficiency and forecasting predictability in 1-, 3- and 6-horizontal months.

Previous studies discussed more on Regression model with robust approach [5] [6]. However, discussions and studies on time series model, AR with bootstrap's hybrid are still scarce compared to other models. Nevertheless, since the bootstrap procedure [7] [8] was introduced, studies involving bootstrap approach has responded to be more encouraging among researches thus caused interest among them to dig up more about this approach especially in the field of time series model such as AR model. This method is as a replacement for recalculations of point estimation by often eliminate observations. [7] [9] [10] [11] supports the use of simple random sampling by replacement for the resampling data that was then called "bootstrap". The empirical evidence [5] [7] [8] [12] [13] [14] [15] [16] suggests that the bootstrap method often gives accurate results. For example, in the study of [12] [17] [18] [19] [20] highlighted the accuracy issue of bootstrap estimation especially in sample mean of statistics.

Bootstrap method is a computer-based method for assessing the accuracy of the statistics [5] [21] [22] [23] [24]. The feasibility using the bootstrap to generate consistent estimates is stated in [25] study, which also suggests that bootstrap is a useful method to calculate the sampling distribution. It has to be said that unlike other measurers of dispersion, the bootstrap confidence intervals and estimation of dispersions are robust to departures from normality and error.

Several years later, [26] [27] has proposed the bootstrap method in his study for a new test of exchange rate profitability's random nature of mechanical trading rules which is also known as the generation of thousands new series for false exchange rates. To test for parametric regression functional form using bootstrap method was proposed by [25] [28] [29] [30]. The Bootstrap method gives a more accurate estimation of the null distribution of the test compared to normal asymptotic theoretical results. 
As mentioned before, there are still only a few studies on the bootstrap approach in AR model. Furthermore, [28] [31] [32] has taken the idea of the bootstrap method in the estimation of the interval of AR models. Moreover, the bootstrap technique is used to allow the uncertainty of parameters while reducing small sample bias in the estimator for the model parameters.

\section{Materials and Methods}

\subsection{Scope of the Research}

In this study the US Dollar (USD) to Malaysian Ringgit (MYR), USD/MYR exchange rate currencies are considered. The exchange rate data used in this study is taken from Bank Negara Malaysia starting from January 2007 until December 2007 which can be referred to in Figure 1 for its daily exchange rates. The main reason of selecting 2007 sample data is due to fluctuative increase of crude oil in that year. According to [33], the year of 2007 was recorded to be the first worse flow period of exchange rate in most Asian countries for the period of 2006 until 2016. This scenario is said to be influenced by the most increased year of crude oil (in USD) that eventually effected the economic growth especially in moderately developed countries, for example Malaysia. Thus, an accurate forecasting for this historical data of exchange rates are essential for the precautionary steps when the crude oil dramatically increases and slows down the economic growth of Malaysia in the future.

Motivated by this issue, the historical exchange rate of USD/MYR is divided into 1-, 3- and 6-horizontal months which involves 227, 186 and 126 data respectively. The selected sample size is motivated by the availability of data during the study. However, this study is limited to determining the appropriate model for the exchange rates currencies of USD/MYR. To be precise, this study only involves the determination of an

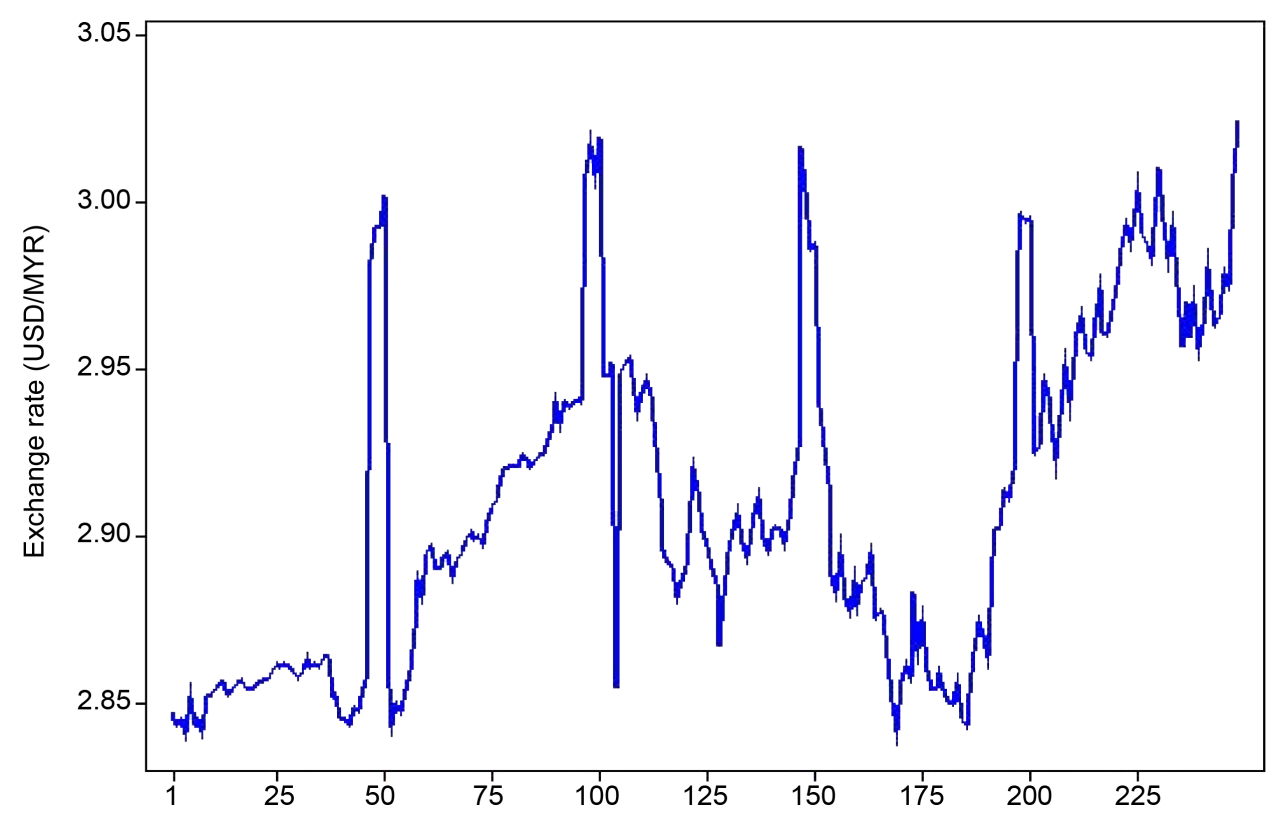

Figure 1. The exchange rate currency of USD/MYR from January until December 2007. 
appropriate forecasting model for USD/MYR exchange rate currencies and does not involve data forecasting.

\subsection{Autoregressive Model}

AR model is about the time series as a linear function of the historical sample data. This model is quite similar to the multiple regression model where the variable of the AR model is regressed with the previous $r_{i}$ value, $r_{i-t}$. The degree of AR model states the total value of the previous movement. The simplest AR model is the first order of autoregressive, AR (1). The general equation of AR model is as follows:

$$
r_{i}=\alpha+\sum_{t=1}^{\rho} \beta_{t} r_{i-t}+\varepsilon_{i}
$$

by neglecting $\alpha$, then

$$
r_{i}=\beta_{1} r_{i-1}+\beta_{2} r_{i-2}+\cdots+\beta_{\rho} r_{i-\rho}+\varepsilon_{i}
$$

for $i=1, \cdots, n$ with $\rho$ is the degree of the AR model and $n$ is the number of data used.

AR model has been widely used in forecasting and predicting of the actual process, based on the analysis discrete and continuous time series. A linear AR model for order $k$ contain adequate statements for the current observation as a combination of previous samples, which was disrupted by some noisy model: $Y_{t}=H\left[Y_{t-1}^{\mathrm{T}} Y_{t-2}^{\mathrm{T}} \cdots Y_{t-k}^{\mathrm{T}}\right]^{\mathrm{T}}+\eta(\mu, \Sigma)$ with $N$ by $k N$ a matrix $H$ which is known as the expected matrix as well as $\eta(\mu, \Sigma)$ as a model for the noise vector.

In general, the $\mathrm{AR}(1)$ process is given by:

$$
\begin{aligned}
Z_{t} & =\varphi Z_{t-1}+a_{t} \\
& =\varphi\left(\varphi Z_{t-2}+a_{t-1}\right)+a_{t} \\
& =\varphi^{2} Z_{t-2}+\varphi a_{t-1}+a_{t} \\
& =\varphi^{2}\left(\varphi Z_{t-3}+a_{t-2}\right)+\varphi a_{t-1}+a_{t} \\
& =\varphi^{3} Z_{t-3}+\varphi^{2} a_{t-2}+\varphi a_{t-1}+a_{t} \\
& =\cdots \\
& =\varphi^{k} Z_{t-k}+\sum_{j=0}^{k-1} \varphi^{j} a_{t-j} \rightarrow \sum_{j=0}^{\infty} \varphi^{j} a_{t-j}
\end{aligned}
$$

If a process does not depend on the future, such as $\operatorname{AR}(1)$ with $|\varphi|<1$, then the process is known as a cause. From AR (1) model, $X_{t}=\varphi X_{t-1}+W_{t}$, where the requirement solution for $X_{t-1}$ is $X_{t-1}=\varphi^{-1} X_{t}+\varphi^{-1} W_{t}=\varphi^{-1} X_{t}+\tilde{W}_{t}$. However, if $|\varphi|>1$, thus the above expression gives stationary representation of $X_{t}$ in the designing the future value of $X_{t}$. Such situation is impossible to be designed because the future is unknown.

\subsection{Bootstrap Method}

Statistics is a science of learning through experience, or in other words, through a learning curve. The statistical theory initiated one of the basic issues that are to express 
the accuracy of a data summary which is part of a process known as statistical inference. Bootstrapping is the latest development techniques to create certain kind of statistical inference. It is a computer-based method to determine the accuracy measure to statistical estimates. Bootstrap was introduced in 1979 as a computer-based method to estimate the standard error of $\theta$. Bootstrapping has the advantage of being completely automatic due to the bootstrap estimates of standard error, that do not require theoretical calculations and is available regardless of how mathematically complicated the estimator [28] [32] [34].

The Bootstrap method is a technique of sampling with replacement. Bootstrap method provides an estimate for any type of statistics such as standard errors, confidence intervals, distribution, etc. Generally, the bootstrap method is being implemented by taking $B$ new samples with replacement from the observed data. Furthermore, the statistics which are to be tested for each new data set is then calculated to obtain bootstrap distribution. The main idea of the bootstrap method is to repeat the sampling of the original data to create, replicate data sets where the features of interest can be assessed. Samples can be taken directly (nonparametric bootstrap) or through the suitability model (parametric bootstrap).

Least-squares estimation method is commonly used to estimate the parameters of a linear model. In some particular assumptions, the least-squares estimator is the best unbiased linear. One of the significant assumptions of the linear model is that the error terms are normally distributed. However, the performance of the least-squares estimator is lower when the error is assumed to be normally distributed. Thus, the parameters of the AR model are estimated by the least squares method without an assumption on the error distribution [35]. The structure of the process allows error resampling and the generation of pseudo data.

One of the ways to deal with this problem is to apply the bootstrap technique, which does not depend on the assumption of normality. The Bootstrap method [4] is a computer-based method to determine the accuracy of measurements for sample estimation. With the Bootstrap method, the estimate for the sample distribution can be performed to almost all statistics.

\subsection{Bootstrapping Concepts}

The Bootstrap method is sampling with replacement from a sample that is to take samples at random from the original sample. Bootstrap sampling depends on the sample itself as just as many sources owned. The principle of bootstrap equality states that the bootstrap estimator for sub sampling (bootstrap method) is equal to the estimated sample. For example, to evaluate the accuracy of $N$ sample data (statistical sample), sampling of $N$ bootstrap is used and hence the statistics from each bootstrap is calculated. The values of bootstrap statistics are used to evaluate the statistics' accuracy of the original statistical samples.

\subsection{Performance Determination of Implementing Bootstrap Method}

The determination of performance for the implementation of bootstrap method is also 
based on comparisons of the statistic forecasting that is RMSE, MAE and MAD. Comparison of the statistical forecasting for the constructed model is carried out to determine the sensitivity of the model. The sensitivity of the model is determined based on the comparison of different outliers. The comparison is between the AR model and BAR model with $1 \%, 5 \%$ and $10 \%$ outliers respectively.

The smallest value of statistical forecast with the increase of outliers indicates a lower sensitivity of the model to outliers. While the larger value of statistical forecast with the increase of outliers indicates a higher sensitivity of the model to outliers. The achievement, determination for the implementation of the AR model with bootstrap approach, BAR will be discussed further in the next discussion on results and findings.

\section{Residual Bootstrap}

There are several bootstrap methods for time series. These include the periodogram bootstrap, block bootstrap and the residual bootstrap [5]. However, it should be emphasized that this study only focused on the residual bootstrap methods for second order AR model, AR (2) with least-square estimator. In general, the residual bootstrap method on AR (2) model begins after the parameter estimation of the AR (2) model by least-square estimator which in turn followed by obtaining the value of the actual residual bootstrap and also the value of the estimated residual bootstrap. The findings of both values of residual bootstrap are then compared through the statistical forecasting.

\section{Residual Bootstrap Algorithm}

In this study, the development of AR models using the bootstrap approach hybrid is proposed. For this purpose, the bootstrap algorithm was developed with the following steps:

\section{Step 1:}

Consider $r_{i}, r_{i-1}, r_{i-2}$ from the original exchange rate data each divided into 1-, 3and 6-horizontal months, which involved 227, 186 and 126 data respectively in the tested exchange rate currencies. From such data, the least square estimation technique was implemented for the estimation of parameters, $\hat{\beta}_{0}, \hat{\beta}_{1}, \hat{\beta}_{2}$ which have been estimated by the method of least square estimation, constant value which is $\hat{r}_{i}=\alpha+\beta_{1} * \beta_{2} * r_{i-2}$ was obtained. Hence, the residue can be obtained by formula $\varepsilon_{i}=r_{i}-\hat{r}_{i}$ where $i=1, \cdots, n$.

\section{Step 2:}

Further, sampling with replacement (bootstrap) was performed on the value of residue, $\varepsilon_{i}$, that was previously obtained to get the value of residual bootstrap, $\varepsilon_{i}^{(j)}$, where $i=1, \cdots, n$ and $j=1, \cdots, B$ where $B=500$, which is the number of bootstrap replications performed. The following are the results obtained:

$$
\varepsilon_{i}^{(j)}=\left(\begin{array}{ccc}
\varepsilon_{1}^{(1)} & \cdots & \varepsilon_{1}^{(500)} \\
\vdots & & \vdots \\
\varepsilon_{n}^{(1)} & \cdots & \varepsilon_{n}^{(500)}
\end{array}\right)
$$




\section{Step 3:}

From the value of residual bootstrap, $\varepsilon_{i}^{(j)}$, which obtained from the previous step, get new data which is $r_{i}^{(j)}=\hat{r}_{i}+\varepsilon_{i}^{(j)}$ where $i=1, \cdots, n$ and $j=1, \cdots, B$. The following are the results obtained:

$$
r_{i}^{(j)}=\left(\begin{array}{ccc}
r_{1}^{(1)} & \cdots & r_{1}^{(500)} \\
\vdots & & \vdots \\
r_{n}^{(1)} & \cdots & r_{n}^{(500)}
\end{array}\right)
$$

\section{Step 4:}

Based on new data obtained before, get an average for the $r_{i}^{(j)}$ to get the value of actual bootstrap, $r_{i}^{B}$, which is $r_{i}^{B}=\left(\sum r_{i}^{(j)} / 500\right)$ where $i=1, \cdots, n$ and $j=1, \cdots, B$. Furthermore, find the average for the value of residual bootstrap $\varepsilon_{i}^{(j)}$, which was obtained in step 2, which is $\varepsilon_{i}^{B}=\left(\sum \varepsilon_{i}^{(j)} / 500\right)$ where $i=1, \cdots, n$ and $j=1, \cdots, B$.

\section{Step 5:}

From the dependent variable $r_{i}^{B}$, the independent variables, $r_{i-1}^{B}$ and $r_{i-2}^{B}$ can be determined.

\section{Step 6:}

Step 1 is repeated to estimate the parameters, which is $\beta_{0}^{*}, \beta_{1}^{*}, \beta_{2}^{*}$.

\section{Step 7:}

Based on the information obtained in the previous steps, obtain the bootstrap estimates, $r_{i}^{B e}$, which is $r_{i}^{B e}=\beta_{0}^{*}+\beta_{1}^{*} * r_{i-1}^{B}+\beta_{2}^{*} * r_{i-2}^{B}+\varepsilon_{i}^{B}$.

Therefore, it can be concluded that the general AR model with hybridize bootstrap approach (BAR) Model can be written as follows: $r_{i}^{*}=\hat{\beta}_{0}^{*}+\sum_{i=1}^{\rho} \hat{\beta}_{t}^{*} r_{i-t}^{*}+\varepsilon_{i}^{*}$. The $\left(^{*}\right)$ sign indicates the model is hybridize bootstrap approach.

\section{Step 8:}

For purposes of comparison in terms of efficiency, sensitivity, accuracy and predictability of the model, get the Root Mean Square Error (RMSE), Mean Absolute Error (MAE) and Median of Absolute Deviation (MAD). The evaluation and comparison of predictions are based on three statistical forecasting [27] as follow:

1) Root mean Square Error (RMSE), $\left\{n^{-1}\left[\sum_{\tau=t+1}^{\tau+n}\left(r_{\tau}-\hat{r}_{\tau}\right)\right]\right\}^{1 / 2}$

2) Mean Absolute Error (MAE), $n^{-1} \sum_{\tau=t+1}^{\tau+n}\left|r_{\tau}-\hat{r}_{\tau}\right|$

3) Median of Absolute Deviation (MAD), median $\left(\left|r_{\tau}-\operatorname{median}\left(r_{\tau}\right)\right|\right)$

where $r_{\tau}$ is an actual value and $\hat{r}_{\tau}$ is the predicted return with time, $\tau$. The result comparison between the uncontaminated original data of the AR model with the uncontaminated original data of the AR model, plus the bootstrap approach is then implemented to show that the BAR approach is more sensitive to outliers than the AR model. Moreover, in terms of forecasting evaluations, the RMSE, MAE and MAD estimation helps to find the best forecasting model between the AR and BAR which shows the most minimum estimation value [36]. The selections of these three formulas are due to the consideration of the estimation theorem which basically concerns the small 
variance in order to have a precise estimation of a model.

\section{Result and Discussion}

In this section, the discussion concerning the effectiveness of the proposed model was performed. The least-squares estimator is widely used, especially in parameter estimation of a model. However, the obvious weakness is that it has a high sensitivity to outliers. Nevertheless, methods to reduce the sensitivity of least-squares estimator to outliers have been identified. The intended method involves the sampling with replacement technique or better known as the bootstrap method [7] [8] [31]. In this study, bootstrap approach will hybridize to AR model which was then known as the bootstrap AR model (BAR). The purpose of using this model is to test the sensitivity of AR model for outliers, the efficiency of the model and forecasting accuracy of the model. Hence the predictability of the model which is based on a comparison made from the results obtained was recognizable.

This section will discuss more about the application of BAR exchange rates, currency of the US Dollar to Malaysian Ringgit, USD/MYR. The forecast model estimation was obtained using the data exchange rate sample data shown in the previous Figure 1. Thus, the estimation value for 1-horizontal months, 3-horizontal months and 6-horizontal month's exchange rates of the US Dollar to Malaysian Ringgit, USD/MYR was shown in Tables 1-3 respectively.

Based on Tables 1-3, the respective sample size of data are 227, 186 and 126. For the robust method, the sample data is considered to cause contamination by $1 \%, 5 \%$ and $10 \%$ for all three horizontals. The bootstrap approach took place after the contaminated

Table 1. 1-Horizontal months exchange rates currency of US Dollar to Malaysia Ringgit (USD/ MYR).

\begin{tabular}{cccc}
\hline & RMSE & MAE & MAD \\
\hline AR $^{\mathrm{a}}$ & 0.295102000 & 0.294506300 & 0.295715600 \\
BAR $(1 \%)^{\mathrm{b}}$ & 0.034463560 & 0.034398430 & 0.034533960 \\
BAR (5\%) & 0.018752940 & 0.017589420 & $\mathbf{0 . 0 1 6 6 9 4 9 8 0}$ \\
BAR (10\%) & $\mathbf{0 . 0 1 6 8 4 0 2 1 0}$ & $\mathbf{0 . 0 1 6 5 1 6 8 6 0}$ & 0.018040160 \\
\hline
\end{tabular}

${ }^{a} \mathrm{AR}$ refer to autoregressive model. ${ }^{\mathrm{b}} \mathrm{BAR}$ refer to bootstrap autoregressive model for different percentages. The bold values are refer to most minimum estimation value of RMSE, MAE and MAD.

Table 2. 3-Horizontal months exchange rates currency of US Dollar to Malaysia Ringgit (USD/MYR).

\begin{tabular}{cccc}
\hline & RMSE & MAE & MAD \\
\hline AR $^{\mathrm{a}}$ & 0.293880800 & 0.293142900 & 0.294708300 \\
$\operatorname{BAR}(1 \%)^{\mathrm{b}}$ & 0.047553280 & 0.047304910 & 0.047365930 \\
$\operatorname{BAR}(5 \%)$ & 0.015749730 & 0.009573013 & $\mathbf{0 . 0 0 7 5 0 7 9 6 4}$ \\
$\operatorname{BAR}(10 \%)$ & $\mathbf{0 . 0 1 1 3 9 2 7 5 0}$ & $\mathbf{0 . 0 0 8 0 7 3 2 5 0}$ & 0.007787416 \\
\hline
\end{tabular}

a, b The abbreviation can be referred to Table 1 . 
Table 3. 6-Horizontal months exchange rates currency of US Dollar to Malaysia Ringgit (USD/ MYR).

\begin{tabular}{cccc}
\hline & RMSE & MAE & MAD \\
\hline AR $^{\mathrm{a}}$ & 0.302655100 & 0.301573800 & 0.303919900 \\
BAR $(1 \%)^{\mathrm{b}}$ & 0.067600590 & 0.067309690 & 0.067981270 \\
BAR (5\%) & 0.022338350 & 0.012197780 & $\mathbf{0 . 0 0 8 8 3 0 6 9 0}$ \\
BAR (10\%) & $\mathbf{0 . 0 3 5 4 8 3 4 2 0}$ & $\mathbf{0 . 0 3 4 9 1 2 5 2 0}$ & 0.039091910 \\
\hline
\end{tabular}

${ }^{\mathrm{a}, \mathrm{b}}$ The abbreviation can be referred to Table 1.

samples data were used in estimating the AR model. The complete procedure of bootstrap can be referred to in section 3.1 where the algorithm of sampling of the residual model of AR with the replacement obtained. Through all procedures, three hybrid models were obtained, namely BAR (1\%), BAR (3\%) and BAR (6\%). Without disregard to the standard AR model, the evaluation of the forecast is made using the equations of RMSE, MAE and MAD in order to find the best model of estimation which literally determines the highest accurate model.

\subsection{Forecasting Accuracy of Model}

Each table shows the performance results of forecasting models for a US Dollar to Malaysian Ringgit, USD/MYR. Tables 1-3 indicate the results for 1-, 3- and 6-horizontal months for forecasting. In each panel, row (2) to (4) shows the measure of forecasting accuracy, RMSE, MAE and MAD. Forecasting accuracy measure which reported in Table 1 shows that a sample performance is varied according to horizontal month's forecast.

For 1-horizontal month in Table 1, the bootstrap approach hybrid to an AR model with $10 \%$ outliers, BAR (10\%), gives the smallest value of statistic forecast compared to an the AR model with the bootstrap approach hybrid at 5\%, BAR (5\%), and bootstrap approach hybrid at $1 \%$, BAR (1\%) outliers. The value obtained with BAR $(10 \%)$ is 0.016840210 and 0.016516860 which are for the value of RMSE and MAE respectively. From Table 2, same goes for 3-horizontal months, which AR model with bootstrap approach hybrid at $10 \%$ outliers, BAR (10\%), giving the smallest value of statistical forecast for RMSE and MAE with 0.011392750 and 0.008073250 respectively. Meanwhile, for MAD statistical forecast, the AR model with bootstrap approach hybrid at 5\% outliers gives the smallest value which is 0.016694980 and 0.007507964 for 1- and 3-horizontal months respectively. From Table 3, for 6-horizontal months, the AR model with bootstrap approach hybrid at $5 \%$ outliers gives the smallest value for all values of statistic forecast, which is $0.022338350,0.012197780$ and 0.008830690 for RMSE, MAE and MAD respectively.

The results obtained indicate the diversity of achievement for each model used are in horizontal month's forecast. However, there are similarities that can be seen in terms of the best model, in order to measure the forecast accuracy for all these three horizontal 
months. The AR model with bootstrap approach hybrid at 10\%, BAR (10\%), and at 5\%, BAR (5\%), outliers are found tend to give smallest value of these three statistical forecasts which makes bootstrap models with $5 \%$ and $10 \%$ outliers is the best for measuring the forecast accuracy for these three horizontal months.

Nevertheless, there are still differences between the values of the result of statistical forecast for BAR (10\%) model and BAR (5\%) model in each 1-, 3- and 6-horizontal months. The value of statistical forecast for BAR (5\%) is greater compared to the value of statistic forecast for BAR (10\%) which explains that the exchange rates, currency of USD/MYR forecast is less accurate for 6-horizontal months. The forecasting reached its maximum accuracy at 3-horizontal months due to the smallest value of forecast accuracy given compared to 1-horizontal months. Thus, forecasting exchange rates, currency of the US Dollar to Malaysian Ringgit is more accurate when tested at 3-horizontal months by using BAR (10\%) model.

\subsection{Forecasting Accuracy of Model}

One of the criteria in the selection of forecasting models is the ability of the proposed model to provide an accurate prediction despite the presence of outliers. The forecasting ability can be measured by identifying the model's sensitivity to outliers that can be identified based on the values of forecast statistics, RMSE, MAE and MAD which indicated with various outliers [37] [38].

There are three levels of outliers involved in this study, which are $1 \%, 5 \%$ and $10 \%$ outliers. Smaller values of forecast statistics with an increase of outliers revealed that the model has a high sensitivity to outliers, thereby enabling this model to be the most appropriate forecasting medium for a tested data.

According to Table 1, the AR model with bootstrap approach hybrid with greater outliers (BAR (10\%), BAR (5\%)), give the smaller value of statistical forecast compared to the AR model with bootstrap approach hybrid with smaller outliers (BAR (1\%)) which rather give greater value of statistical forecast. This can be seen in the three horizontal months, which is obviously produces to give the smallest value even with the increase of outliers.

For 1- and 3-horizontal months, the AR model with bootstrap approach hybrid at $10 \%$, BAR (10\%), outliers revealed the smallest value of statistical forecast for RMSE and MAE, followed by the AR model with bootstrap hybrid at 5\%, BAR (5\%), outliers then followed by the AR model with bootstrap approach hybrid at $1 \%$, BAR (1\%) outliers which gives greatest value among all three models with outliers. Similarly to the 6-horizontal months data which also gives the smallest value of forecast statistic; RMSE, MAE and MAD, even on the AR model with bootstrap approach hybrid at 5\%, BAR (5\%) outliers, but still it produces the smaller value compared to model at $1 \%$ outliers, BAR (1\%).

Overall, the AR model with the bootstrap approach hybrid, BAR, which has a greater outliers is more sensitive to the presence of extreme data that could further improve the forecasting accuracy, especially for those that is very risky to have extreme data. 


\subsection{The Efficiency of Model}

A developmental model is best when it fulfills the estimation's criteria which is known as Best, Linear, Unbiased, Efficient, or in short, BLUE. In addition, the sensibility of a model which is closely related to the sensitivity of the model to outliers need to be considered. Therefore, the bootstrap approach hybrid on the AR model is developed. The bootstrap model has a high sensitivity to outliers. This can be proved by the smaller values of statistic forecast even with the increase in the percentage of contaminated data.

Therefore, it can be concluded that the AR model with the bootstrap approach is more efficient than the AR model itself. Thus, the developed bootstrap model can be applied as a forecasting model for exchange rate data.

\subsection{Predictability of Exchange Rates}

Research has shown that the exchange rates' predictability is interrelated with the forecasting accuracy which can be measured by the model used in [38]. The forecasting accuracy can be seen in the obtained forecast statistic when the data are tested with BAR models, BAR (1\%), BAR (5\%) and BAR (10\%). The smaller the values obtained, the more accurate the forecast. Thus, the estimated values will be close to the actual value.

Predictability of exchange rates can be determined by comparing the AR model and BAR model through the values of statistics forecast; RMSE, MAE and MAD. From the previous discussion, it clearly shows the AR model with the bootstrap approach hybrid, BAR (1\%), BAR (5\%) and BAR (10\%) has a higher forecast accuracy than the AR model.

For the exchange rates of Malaysian Ringgit to US Dollar (USD/MYR), the predictability is based on the comparison shown in Tables 1-3. The values of forecast statistics vary according to horizontal months. However, the values of forecast statistic shown by the three proposed models are smaller compared to the values of forecast statistic when using an AR model. This can be seen in the results for the 3-horizontal months in Table 2 that is the values of forecast statistics; RMSE, MAE and MAD. BAR model with BAR (1\%), BAR (5\%) and BAR (10\%) each give values 0.047553280, 0.047304910, $0.047365930 ; 0.015749730,0.009573013,0.007507964$ and $0.011392750,0.008073250$, 0.007787416 respectively.

Through comparison with the values of the forecast statistics; RMSE, MAE and MAD obtained for the AR model, each with $0.293880800,0.293142900$ and 0.294708300 respectively, it clearly shows that the forecast values of the proposed model are smaller. This proved that the predictability of the exchange rates is better by using the BAR model because it can result in more accurate forecast statistics in which it can acquire even the smallest of errors, even with the increase of outliers.

\section{Conclusion}

In this study, the AR model was used. However, this model is sensitive to outliers. So, in order to alleviate this problem, the BAR model was proposed. The bootstrap approach was hybridized to the AR model, and produced a new model known as the BAR model. The results revealed that the proposed model has improved the forecasting ac- 
curacy, reduced the sensitivity of the AR model to outliers, and increasing the efficiency of the forecasting model. It can be proven by the comparison of the BAR model and AR model at $1 \%, 5 \%$ and $10 \%$ outliers through the smaller values of RMSE, MAE and MAD. From the results obtained, the proposed model has improved the AR model. This condition, thus also improved the level of predictability of the AR model on the tested exchange rate currencies.

\section{Acknowledgements}

The authors would like to acknowledge the financial support from the Graduate School of Universiti Malaysia Terengganu (UMT) through the Graduate Financial Scheme (GFS), and also the School of Informatics and Applied Mathematic (SIAM), as well as the Research Management Centre (RMC) of University Malaysia Terengganu for their continuous and diligent support.

\section{References}

[1] Copeland, L. (2005) Exchange Rates and International Finance. Prentice-Hall, New York.

[2] Grubbs, F.E. (1969) Procedures for Detecting Outlying Observations in Samples. Technometrics, 11, 1-21. http://dx.doi.org/10.1080/00401706.1969.10490657

[3] Owen, A.B. and She, Y. (2012) Outlier Detection Using Nonconvex Penalized Regression. Journal of the American Statistical Association, 106, 626-639.

[4] Moosa, I. (2008) Forecasting the Chinese Yuan-US Dollar Exchange Rate under the New Chinese Exchange Rate Regime. International Journal of Business and Economics, 7, 23-35. https://www.researchgate.net/publication/228425338_Forecasting_the_Chinese_Yuan-US_ Dollar_Exchange_Rate_under_the_New_Chinese_Exchange_Rate_Regime

[5] Mokhtar, A. (1990) On a Robust Correlation Coefficient. The Statisticia, 39, 455-460. http://dx.doi.org/10.2307/2349088

[6] Efron, B. and Tibshirani, R.J. (1993) An Introduction to the Bootstrap. Chapman \& Hall, New York. http://dx.doi.org/10.1007/978-1-4899-4541-9

[7] Efron, B. (1979) Bootstrap Methods: Another Look at the Jackknife. The Annals of Statistics, 7, 1-26. http://dx.doi.org/10.1214/aos/1176344552

[8] Efron, B. (1982) The Jackknife, the Bootstrap and Other Resampling Plans. CBMS-NSF Regional Conference Series in Applied Mathematics, Monograph 38, SIAM, Philadelphia. http://dx.doi.org/10.1137/1.9781611970319

[9] Fernandez, J.M.V. and Manteiga, W.G. (2000) Resampling for Checking Linear Regression Models via Non-Parametric Regression Estimation. Computational Statistics and Data Analysis, 35, 211-231. http://dx.doi.org/10.1016/S0167-9473(99)00117-6

[10] Wu, C.F.J. (1986) Jackknife, Bootstrap and other Resampling Methods in Regression Analysis. The Annals of Statistics, 14, 1261-1295. http://dx.doi.org/10.1214/aos/1176350142

[11] Thombs, L.A. and Schucany, W.R. (1990) Bootstrap Prediction Intervals for Autoregression. Journal of the American Statistical Association, 85, 486-492. http://dx.doi.org/10.1080/01621459.1990.10476225

[12] Bickel, P.J. and Freedman, D.A. (1981) Some Asymptotic Theory for the Bootstrap. The Annals of Statistics, 9, 1196-1217. http://dx.doi.org/10.1214/aos/1176345637

[13] Freedman, D. and Peters, S. (1984) Some Note on the Bootstrap in Regression Problems, 
Journal of Economic Statistics, 2, 406-407.

[14] Freedman, D. and Peters, S. (1984) Bootstrapping a Regression Equation: Some Empirical Results. Journal of the American Statistical Association, 79, 97-106. http://dx.doi.org/10.1080/01621459.1984.10477069

[15] Freedman, D. and Peters, S. (1984) Bootstraping an Econometric Model: Some Empirical Results. Journal of Business Economic Statistics, 2, 150-158.

[16] Efron, B. and Tibshirani, R.J. (1993) An Introduction to the Bootstrap. Chapman \& Hall, New York.

[17] Singh, K. (1981) On the Asymptotic Accuracy of Efron's Bootstrap. The Annals of Statistics, 9, 1187-1195. http://dx.doi.org/10.1214/aos/1176345636

[18] Beran, R. (1982) Estimated Sampling Distributions: The Bootstrap and Competitors. The Annals of Statistics, 10, 212-225. http://dx.doi.org/10.1214/aos/1176345704

[19] Pasquariello, P. (2002) Uncertainty of Trading Rules in Accuracy Markets: An Application of Non-Parametric Bootstrapping. Journal of Multinational Financial Management, 12, 107-133. http://dx.doi.org/10.1016/S1042-444X(01)00049-4

[20] Brzozowska-Rup, K. and Orlowski, A. (2004) Application of Bootstrap to Detecting Chaos in Financial Time Series. Physica A, 344, 317-321. http://dx.doi.org/10.1016/j.physa.2004.06.142

[21] Bustors, O.H. and Yohai, V.J. (2012) Robust Estimates for ARIMA Models. Journal of the American Statistical Association, 81, 155-168. http://dx.doi.org/10.1080/01621459.1986.10478253

[22] Muhamad Safiih, L., Mohd Fadli, H., Nurul Hila, Z. and Mohd Noor Afiq, R. (2016) Estimating the New Keynesian Model by Bootstrap Method for Johor Economy Tourism. Modern Economy, 7, 1061-1069. http://dx.doi.org/10.4236/me.2016.710108

[23] Lola, M.S. and Zainuddin, N.H. (2016) The Performance of Double Bootstrap Method for Large Sampling Sequence. Open Journal of Statistics, 6, 805-813. http://dx.doi.org/10.4236/ojs.2016.65066

[24] Lola, M.S., Alwi, W.S.W. and Zainuddin, N.H. (2016) Sample Selection Model with Bootstrap (BPSSM) Approach: Case Study of the Malaysian Population and Family Survey. Open Journal of Statistics, 6, 741-748. http://dx.doi.org/10.4236/ojs.2016.65060

[25] Brownstone, D. (1990) Bootstrapping Improved Estimators for Linear Regression Models. Journal of Econometrics, 44, 171-187. http://dx.doi.org/10.1016/0304-4076(90)90078-8

[26] Levich, R.M. and Thomas, L.R. (1993) The Significance of Technical Trading-Rule Profits in the Foreign Exchange Market: A Bootstrap Approach. Journal of International Money and Finance, 12, 451-474. http://dx.doi.org/10.1016/0261-5606(93)90034-9

[27] Ko, H.H. and Ogaki, M. (2015) Granger Causality from Exchange Rates to Fundamentals: Hat Does the Bootstrap Test Show Us? International Review of Economics and Finance, 38, 198-206. http://dx.doi.org/10.1016/j.iref.2015.02.016

[28] Konietschke, F., Bathke, A.C., Harrar, S.W. and Pauly, M. (2015) Parametric and Nonparametric Bootstrap Methods for General MANOVA. Journal of Multivariate Analysis, 140, 291-301. http://dx.doi.org/10.1016/j.jmva.2015.05.001

[29] Whang, Y.J. (2000) Consistent Bootstrap Test of Parametric Regression Functions. Journal of Econometrics, 98, 27-46. http://dx.doi.org/10.1016/S0304-4076(99)00078-0

[30] Berg, A., Paparoditis, E. and Politis, D.N. (2010) A Bootstrap Test for Time Series Linearity. Journal of Statistical Plan and Inference, 140, 3841-3857. http://dx.doi.org/10.1016/j.jspi.2010.04.047 
[31] Clements, M.P. and Taylor, N. (2001) Bootstrapping Prediction Intervals for Autoregressive Models. International Journal of Forecasting, 17, 247-267. http://dx.doi.org/10.1016/S0169-2070(00)00079-0

[32] Fu, K.A., Li, Y. and Ng, A.C.Y. (2013) Asymptotic for the Residual-Based Bootstrap Approximation in Nearly Nonstationary AR(1) Models With Possibly Heavy-Tailed Innovations. Statistics \& Probability Letters, 83, 2553-2562.

http://dx.doi.org/10.1016/j.spl.2013.07.006

[33] Hussain, M., Zebende, G.F., Bashir, U. and Donghong, D. (2017) Oil Price and Exchange Rate Co-Movements in Asian Countries: Detrended Cross-Correlation Approach. Physica A, 46, 338-346. http://dx.doi.org/10.1016/j.physa.2016.08.056

[34] Ruppert, D. (2004) Statistics and Finance: An Introduction. Springer, New York. http://dx.doi.org/10.1007/978-1-4419-6876-0

[35] Bose, A. (1988) Edgeworth Correction by Bootstrap in Autoregressions. The Annals of Statistics, 16, 1709-1722. http://dx.doi.org/10.1214/aos/1176351063

[36] Qi, L. and Suojin, W. (1998) A Simple Consistent Bootstrap Test for a Parametric Regression Function. Journal of Econometrics, 87, 145-165. http://dx.doi.org/10.1016/S0304-4076(98)00011-6

[37] Gupta, S. and Kashyap, S. (2016) Modelling Volatility and Forecasting of Exchange Rate of British Pound Sterling and Indian Rupee. Journal of Modelling in Management, 11, 389404. http://dx.doi.org/10.1108/JM2-04-2014-0029

[38] Preminger, A. and Franck, R. (2007) Forecasting Exchange Rates: A Robust Regression Approach. International Journal of Forecasting, 23, 71-84.

http://dx.doi.org/10.1016/j.ijforecast.2006.04.009

Submit or recommend next manuscript to SCIRP and we will provide best service for you:

Accepting pre-submission inquiries through Email, Facebook, LinkedIn, Twitter, etc. A wide selection of journals (inclusive of 9 subjects, more than 200 journals)

Providing 24-hour high-quality service

User-friendly online submission system

Fair and swift peer-review system

Efficient typesetting and proofreading procedure

Display of the result of downloads and visits, as well as the number of cited articles

Maximum dissemination of your research work

Submit your manuscript at: http://papersubmission.scirp.org/

Or contact ojs@scirp.org 